\title{
Thermal wave interferometry measurements and microstructural analysis of twin wire arc spray cylinder coatings for passenger car engines
}

\author{
Jannik Arbogast ${ }^{1,2}$, Rolf Schaller ${ }^{1}$, Sören Lindemann ${ }^{1}$, Bernd Schilder ${ }^{1}$, Magnus Rohde ${ }^{2}$, \\ Hans Jürgen Seifert ${ }^{2}$ \\ Jannik Arbogast, Mercedes-Benz AG, Mercedesstraße 140, 70327 Stuttgart, Germany
}

\begin{abstract}
A B S T R A C T
With intent to increase the efficiency of combustion engines in passenger cars, thermally sprayed cylinder bore coatings replaced cast iron liners as cylinder running surfaces over the past years. Since the functionality of an engine depends on the life-long integrity of these coatings only a few $100 \mu \mathrm{m}$ thick, monitoring their physical properties in series production is most important. This contribution focuses on the non-destructive testing of thermally sprayed coatings by thermal wave interferometry (TWI), investigating the relationship between thermal and microstructural properties. Microstructural characteristics such as laterally expanded splat interfaces or spherical pores correlate with macroscopic quality criteria such as adhesion, cohesion, or corrosion resistance. Further on, especially splat interfaces act as thermal barriers within the coatings. Hence, TWI thermal diffusivity measurements were compared to optical microscopy images of polished cross sections and threedimensional X-ray computed tomography data at multiple positions in a crankcase. Both, microscopy images as well as computed tomography data were analyzed quantitatively by image processing algorithms. Due to this, local variations in thermal diffusivity were found, which revealed a significant correlation with microstructural characteristics. Further on, a significant influence of the amount, size, and shape of microstructural volume defects on the local thermal diffusivity of thermally sprayed coatings is carried out in this contribution.
\end{abstract}

Keywords:

Thermal wave interferometry

Thermal diffusivity

Twin wire arc spraying

Microstructural analysis

Cross section microscopy

X-ray computed tomography

\section{Introduction}

In order to reduce emissions and fuel consumption, as well as to fulfill the rising requirements and regulation concerning $\mathrm{CO}_{2}$ emissions, it is an important objective of the automotive industry to enhance the efficiency of modern powertrains in the near future. In this context, thermally sprayed cylinder bore coatings have proven to reduce not only the weight but also the frictional losses of combustion engines. Mercedes Benz developed the NANOSLIDE ${ }^{\mathrm{TM}}$ process chain, which combines twin wire arc spraying with a surface finish through honing. Due to this technology, friction resistance losses between pistons and cylinder running surface is reduced by up to $50 \%[1,2]$. Durability and life long integrity of thermally sprayed cylinder bore coatings are two mandatory aspects concerning the functionality of combustion engines and depend among others on the coatings' mechanical properties and microstructural characteristics. Binding errors, splat interfaces, and pores define decisive defects of the material influencing its' mechanical properties, which may be influenced by thermal spray parameters $[3,4]$. In terms of process monitoring a non destructive and non contact evaluation of these features with a short feedback loop is highly de sired. By now, only destructive off site testing is carried out by optical microscopy of polished cross sections or by high resolution computed tomography of material samples. However, several publications have shown that thermal properties of thermally sprayed coating layers can be directly correlated to their microstructural composition, enabling to use thermal measurements to inversely access the coatings' micro structural properties non destructively [4 7]. While optically and non optically excited thermography opens a broad spectrum of possible application [8], laser flash analysis is an established method to measure thermal diffusivities. Since commercially available systems require planar samples with a standardized size and shape as well as access to both sides of the material [9], these are not feasible for the investiga tion of rather thin and curved thermally sprayed cylinder bore coatings.

An alternative method to investigate thermal diffusivities of a coated substrate with only one side access to the specimen is called thermal wave interferometry (TWI) [10]. TWI has been established for a wide range of characterization and testing applications during the past decades [11 13 . The measuring principle is based on propagation,

\footnotetext{
E-mail address: jannik.arbogast@daimler.com (J. Arbogast).

${ }^{1}$ Mercedes-Benz AG, Stuttgart, Germany.

${ }^{2}$ Karlsruhe Institute of Technology, Karlsruhe, Germany.
} 
reflection, and interference of thermal waves, which are determined by the material's thermal diffusivity as well as the coating thickness. Surface heating with a sinusoidal amplitude modulated heat source may trigger such thermal waves in a specimen [14]. While infrared sensors are capable of detecting the material's thermal response, an additional lock in amplifier allows to discriminate between its' ampli tude and phase information. Laser excited lock in thermography is a specific form of TWI and provides access to the phase shift between surface temperature and sinusoidal amplitude modulated excitation. Thus, it allows to estimate the material's thermal diffusivity and even gathers information about its' local variations when combined with a fast, two dimensional infrared detector.

In this study, thermal diffusivity measurements of twin wire arc sprayed cylinder bore coatings are performed using laser excited lock in thermography. The resulting information are compared to the local microstructural characteristics depicted by optical microscopy images of polished cross sections as well as three dimensional $\mathrm{X}$ ray computed tomography data of material samples. The investigations aim to find an approach for non destructive, non contact, fast, and reproducible eva luation of thermally sprayed coatings.

\section{Materials - NANOSLIDE ${ }^{\mathrm{TM}}$}

The production process of Mercedes Benz engines with thermally sprayed cylinder running surfaces as well as the technology itself is called NANOSLIDE ${ }^{\mathrm{TM}}$. The process chain starts with casting and thermal as well as mechanical treatments of aluminum crankcases. Furthermore, it includes roughening the cylinder bore, followed by twin wire arc spraying, mechanical machining, and honing of low alloyed carbon steel coatings. By mechanically roughening the cylinder bores using tools with defined cutting edges prior to the coating, a rough ening profile with defined as well as non defined macro and micro structures are created, which are crucial to achieve the required ad hesion between the layer and substrate [1]. The roughened profile shows extensions of about $150 \mu \mathrm{m}$.

Twin wire arc spraying is realized by a rotating torch, which is plunged into the cylinder bores while the coating material is carried into the torch via two wires. An electrical voltage applied to the wires causes an arc and melts the coating material [15]. Process gas is used to accelerate and spray the molten particles onto the roughened cylinder bore surface and moreover to form a protective atmosphere against oxidation during the spray process. Since hot, molten particles hit the substrate and cool down consecutively, a typical lamellar structure of partially separated splats and porosity builds up [4,16 18]. Fig. 1 shows a typical morphology of thermally sprayed coating including porosity and lateral splat interfaces. Additionally, to avoid contamina tion of the cylinder coating due to excessive particles and to lower the oxygen content within the spray jet, the atmosphere within the crank case is extracted continuously during the coating process. Computa tional fluid dynamic (CFD) simulations of the complex gas flow during the coating process as well as coating experiments have shown that the mass fraction of Oxygen in the atmosphere surrounding the spray jet strongly influences the lamellar structure [3]. Higher mass fraction of Oxygen leads to enhanced oxidation of the spray particles and therefore increases the amount of splat interfaces in the coating. If Oxygen is banned almost entirely during the coating process, rather dense ther mally sprayed coatings with very few splat interfaces may appear $[3,4,18]$.

Finally, multiple honing steps are performed to create the required surface structure of the cylinder coating for latter operation in the combustion engine, while finish honed cylinder bore coatings exhibit material thicknesses of a few $100 \mu \mathrm{m}$.

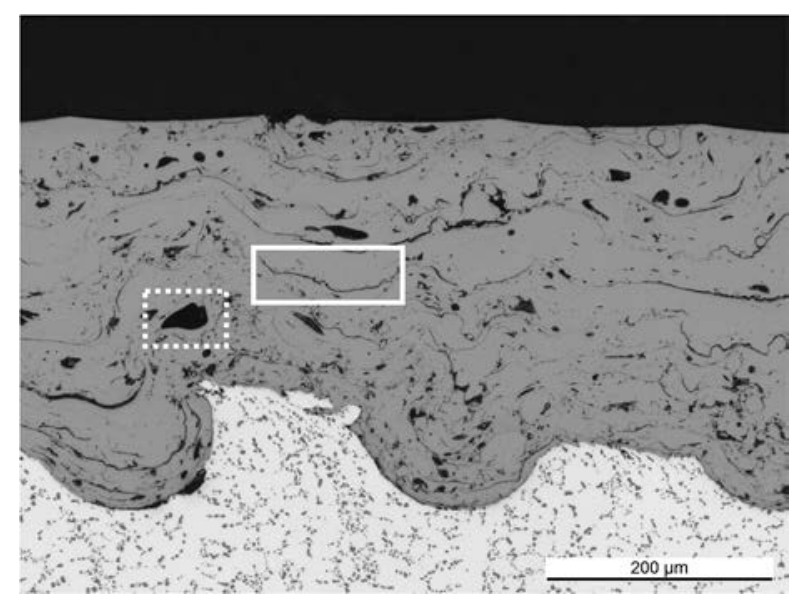

Fig. 1. Optical microscopy images of a cross section from a cylinder bore coating at position $10 \mathrm{~mm}$. The functional cylinder coating is shown in dark gray, while the crankcase aluminum alloy is depicted in light gray. The substrate shows the mechanical roughening profile, which enhances the adhesion of the coating to the substrate. The cross section shows the typical morphology of thermally sprayed coatings including lateral splat interfaces and enhanced porosity. The embedding material is shown in black. Solid box: lateral splat interface; dotted box: spherical pore.

\section{Thermal wave interferometry (TWI)}

\subsection{Theory}

Characteristics of thermally sprayed coatings such as the thermal diffusivity $\alpha$ can be measured by thermal wave interferometry [19 21]. Based on the heat conduction equation

$\frac{\partial T}{\partial t}=\alpha \nabla^{2} T$

TWI uses a periodically modulated heat source with frequency $f$ to generate a temperature oscillation $T(f)$ on the surface of a specimen. The heat conduction Eq. (1) can be solved using a wave approach, re sulting in heavily damped so called 'thermal waves' propagating dif fusively from the surface through the coating [14]. Thermal waves may be used to investigate thermal and mechanical properties of all kinds of materials non destructively as well as contactless. If thermal waves are generated in a layered sample with different thermal properties of layer and substrate, they may be partially reflected at the interface of layer and substrate, which can be compared to the reflection of magnetic or ultrasonic waves [22]. The reflection coefficient $R_{x, y}$ represents the different material properties of coating and substrate and depends on the thermal effusivities $\epsilon$ as

$R_{x, y}=\frac{\epsilon_{x}-\epsilon_{y}}{\epsilon_{x}+\epsilon_{y}}=\frac{\rho_{x} c_{x} \lambda_{x}-\rho_{y} c_{y} \lambda_{y}}{\rho_{x} c_{x} \lambda_{x}+\rho_{y} c_{y} \lambda_{y}}$

in which $\rho$ is the density, $c$ is the specific heat and $\lambda$ is the thermal conductivity, while $x$ symbolizes the coating and $y$ the substrate. The reflected waves lead to interference effects with the incoming thermal waves causing a phase shifted temperature modulation of the surface temperature compared to the incoming modulated heat from the source [20]. Based on thermal and mechanical properties of the layer and substrate of the sample, the surface temperature $T(f)$ in a one dimen sional approach was first described by Bennett and Patty [14] as

$T(f) \propto \frac{1+R \cdot \exp (-2 \cdot(1+i) \cdot d / \mu)}{1-R \cdot \exp (-2 \cdot(1+i) \cdot d / \mu)}$

The thermal diffusion length $\mu=\sqrt{\alpha / \pi f}$ depends on the thermal dif fusivity $\alpha$ of the sample as well as the modulation frequency $f$ of the heat source, which allows to obtain information from different depths 
of the sample by varying the modulation frequency of the thermal wave. The thickness of the coating material is described by $d$. If the material thickness has the same order of dimension as the thermal diffusion length $\mu$, thermal information of the coating such as the thickness itself or inner volume defects can be obtained via TWI [10]. Consequently, if thermal diffusion lengths are chosen lower than the material thickness, thermal waves may not reach the layer substrate interface and the layer material can be seen as bulk material.

The thermal diffusivity $\alpha$ is connected to the density $\rho$, the specific heat capacity $c$ and the thermal conductivity $\lambda$ as

$\alpha=\frac{\lambda}{\rho \cdot c}$

The surface temperature in (3) can be separated into amplitude and phase, while the phase shift $\phi(f)$ between temperature modulation of the surface temperature compared to the incoming modulated heat from the source may be written as

$\phi(f)=\tan ^{-1}\left(\frac{-2 R \cdot \exp (-2 \eta \sqrt{f}) \cdot \sin (2 \eta \sqrt{f})}{1-(R \cdot \exp (-2 \eta \sqrt{f}))^{2}}\right)-\frac{\pi}{4}$

in which $\eta=d \cdot \sqrt{\pi / \alpha}$ is proportional to the coating's thickness and the square root of the inverse thermal diffusivity [14]. Consequently, thermal diffusivity measurements via TWI can only be obtained if the layer thickness $d$ is measured with a reference method. Since the main emphasis of this work is to measure thermal diffusivities of cylinder bore coatings, $X$ ray computed tomography data (Section 4.2) was used to evaluate the respective material thickness of the coatings.

\subsection{Lock In System}

Thermal wave interferometry measurements were performed on a laser excited lock in thermography system by edevis $\mathrm{GmbH}$ (Stuttgart, Germany) including a high power diode laser DSC11 (OsTech e.K., Berlin, Germany) with $250 \mathrm{~W}$ maximum power and $938 \mathrm{~nm}$ wavelength. A sinusoidal laser amplitude is modulated by a signal generator and transferred onto the sample surface via optical fiber and dichroic mirror. Shape and size of the laser beam are controlled by a lens system, which is placed right at the optical fiber's end. The laser beam is chosen to have a top hat shape. The lock in system also contains an infrared camera FLIR X6580 sc (FLIR Systems, Wilsonville, USA), which is synchronized with the excitation source via the signal generator module and has an InSb detector with $640 \times 512 \mathrm{px}$, allowing for a maximum frame rate of $355 \mathrm{~Hz}$ at maximum window size but also for fast frame rates up to $600 \mathrm{~Hz}$ at smaller subwindows. The described setup uses an optical resolution of $0.2 \mathrm{~mm}$ per camera pixel. The peak of the infrared detector's spectral sensitivity is around $5800 \mathrm{~nm}$ wavelength. To achieve a coaxial set up of laser beam and infrared camera, the dichroic mirror is reflective for wavelengths lower than $1200 \mathrm{~nm}$, such that the optical fiber end is placed at an angle of $90^{\circ}$ to the measured sample. Since the dichroic mirror is transmissive for wavelengths larger than $1200 \mathrm{~nm}$, the infrared camera is placed behind the mirror, in line to the measured sample. The laser beam excitation is synchronized with the frame rate of the camera, such that the camera frame rate sends a time signal to the signal generator. Based on the model described in Section 3.1, the infrared camera detects a phase shifted temperature oscillation on the coating surface compared to the excitation of the laser beam. A Fast Fourier Transformation (FFT) is used to calculate amplitude and phase from the detected temperature oscillations. In order to investigate the thermal diffusivity of cylinder bore coatings in the crankcase non de structively, laser beam and detected radiation are deflected by another $90^{\circ}$ within the cylinder bore using a gold plated aluminum mirror (Edmund Optics, Barrington, USA), which is plunged into the crank case. The entire lock in thermography system can be found in Fig. 2.

TWI measurements were performed with a laser spot diameter on the sample surface of $20 \mathrm{~mm}$ and total power of $125 \mathrm{~W}$ resulting in a

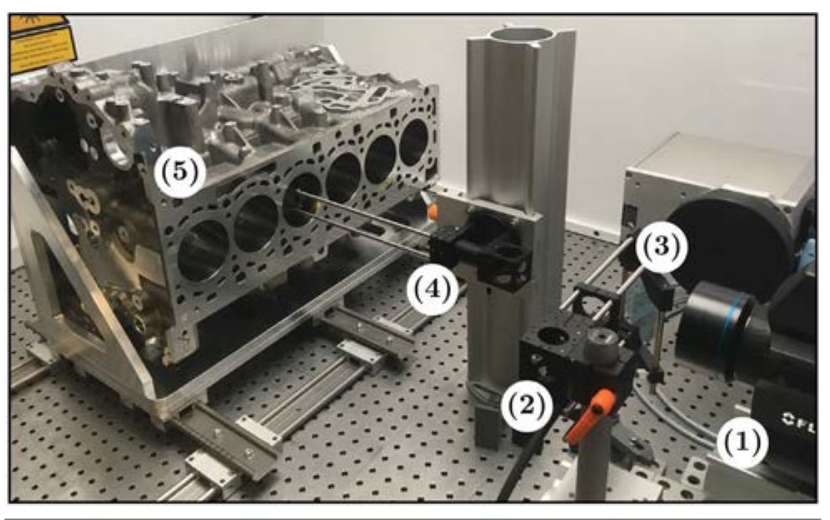

(5) Combustion engine

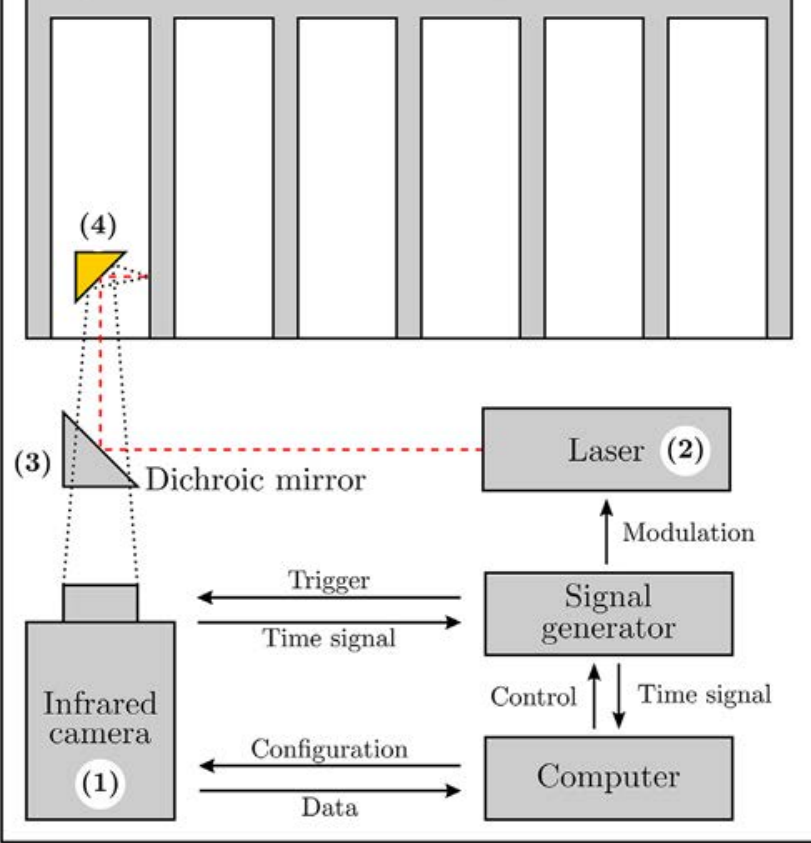

Fig. 2. Photography and sketch of the laser-excited lock-in thermography system. (1) Infrared camera, (2) laser fiber end, (3) dichroic mirror, (4) goldplated aluminum mirror including attachment and (5) combustion engine of type OM656 (Mercedes-Benz AG, Stuttgart, Germany).

power density of about $0.1 \mathrm{~W} / \mathrm{mm}^{2}$. Acquired TWI measurements of the coatings are averaged over a spot size of $6.7 \mathrm{~mm}$. The excitation fre quency $f$ was set to $5,6,10,15,20,25,30,40,50,60,75,100,120$ and $150 \mathrm{~Hz}$. The surface temperature of the cylinder coating is measured $5 \mathrm{~s}$ for each excitation frequency. However, well known systematic errors during lock in thermography result from lock in measurements during early non linear heating of the sample [23]. To avoid non linear sample heating during the actual measurements, the cylinder coating is pre heated in advance of each measurement for additional $5 \mathrm{~s}$ at each ex citation frequency. Preliminary experiments have shown, that this time scale is sufficient to eliminate non linear heating effects for all applied excitation frequencies. Lower excitation frequencies than $5 \mathrm{~Hz}$ were chosen to be unreasonable, since lateral heat diffusion could not be neglected anymore. The use of a one dimensional heat diffusion model as described in Section 3.1 is only valid, if the thermal diffusion length $\mu$ is much smaller than the beam size [19]. As found in Section 5.1, thermal diffusion lengths range between $105 \mu \mathrm{m}$ and $777 \mu \mathrm{m}$, which is comparably small to the excitation area on the sample surface of about $20 \mathrm{~mm}$. Higher frequencies were limited by hardware properties. Phase values obtained at various modulation frequencies are used to fit the 
Cylinder head sealing surface

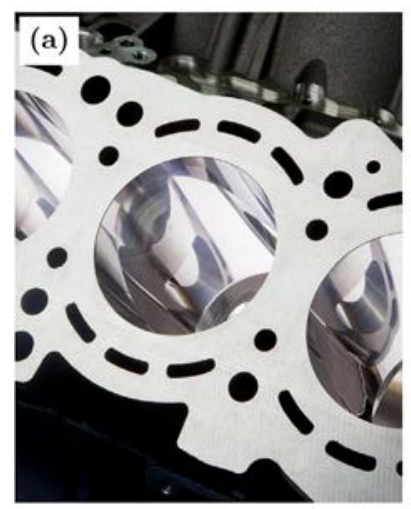

defects in the cross section image of the cylinder coating, while a lateral splat interface is marked by the solid box and a pore is marked by the dotted box.

Samples for metallographic cross section (Section 4.1) and and CT measurements (Section 4.2) were cut out off one cylinder liner of one randomly selected engine. The measurement positions equal TWI measurement positions in Section 3.2.

\subsection{Cross sections}

Microstructural analysis of the thermally sprayed coatings was achieved using optical microscopy images, taken with an Axio Observer microscope (Carl Zeiss AG, Oberkochen, Germany). Therefore, polished cross sectional specimen were cut out of the cylinder bores vertically with $20 \mathrm{~mm}$ length at the described positions (Fig. 3) followed by multiple preparation steps. Since single microscopy images show sizes of about $650 \mu \mathrm{m} \times 650 \mu \mathrm{m}$, the entire cross sections are covered by around 30 microscopy images. The quantitative microscopy image analysis was carried out using an algorithm based on OpenCV im plemented in Python, which focuses on lateral splat interfaces in the cross sectional image data, which were processed by global thresh olding segmentation and connected component labeling [24]. Latter enables access to a large variety of features of the detected impurities in the coating such as individual defect size or minor and major axis lengths. The analysis algorithm filters the detected labels based on their shape and only processes interfaces showing lateral orientation and distinct extension based on the evaluated axis of the splat. Afterwards, the detected area of the processed lateral splat interfaces is evaluated regarding the percentage of the detected interfaces to the coating ma terial. While each single image is processed by the analysis algorithm, mean segmented splat area and its' standard deviation of each cross section are calculated based on the analysis of the 30 individual mi croscopy images per cross section.

\section{2. $X$ ray computed tomography}

$\mathrm{X}$ ray computed tomography (CT) is used to obtain three dimen sional information about inner volume defects in thermally sprayed cylinder bore coatings. Therefore, specimens with a size of $5 \times 5 \times 5 \mathrm{~mm}$ were cut out next to the cross section specimens and measured by a Nanofocus CT system (General Electric, Boston, USA). The cathode current of the CT system was chosen as $100 \mu \mathrm{A}$ and the acceleration voltage as $160 \mathrm{kV}$. The data was acquired with $3750 \mathrm{~ms}$ integration time per detector pixel and overall 1800 projections for $360^{\circ}$ rotation of the specimens. The three dimensional data set is trans formed into a two dimensional image stack parallel to the cylinder running surface. The volume data contain information about local mi crostructural variations, pore size and position distribution as well as material thickness of the coating. The quantitative image analysis of CT data uses high pass filtering, locally adaptive segmentation with a constant, quadratic window size and connected component labeling to identify microstructural variations within the thermally sprayed used to evaluate the microstructural properties of the thermally sprayed cylinder bore coatings. These two methods were taken to identify me chanical defects within the material and analyze them quantitatively. Two basic defects are defined, splat interfaces and pores. Splat inter faces are laterally orientated defects, showing a distinct horizontal ex tension with an aspect ratio $a$ of major and minor axis larger than 3, while pores are defined as almost spherical defects without distinct orientation and extension $(a<3)$ [5]. Fig. 1 does show both types of

Table 1

TWI measurements, cross section analysis and CT analysis of cylinder coatings at different measurement heights along the liner of the cylinder crankcase. The indicated positions are described from the cylinder head sealing surface towards the crankshaft space at the bottom of the engine (Fig. 1). All shown TWI values represent mean values summarizing the measurements of three engines and the corresponding six cylinder liners, while the given uncertainties represent the standard deviation. Further, uncertainties of the segmented splat area, porosity and mean pore size represent the standard deviation based on the analysis of 30 individual microscopy images per cross section and a two dimensional image stack (CT data) at each measurement position.

\begin{tabular}{|c|c|c|c|c|c|c|}
\hline Position [mm] & $R$ & $\eta\left[10^{-3} \mathrm{~s}^{1 / 2}\right]$ & $\alpha\left[\mathrm{mm}^{2} / \mathrm{s}\right]$ & Seg. splat area $[\%]$ & Porosity [\%] & Mean pore size $\left[\mu \mathrm{m}^{3}\right]$ \\
\hline 10 & $-0.34 \pm 0.01$ & $0.20 \pm 0.01$ & $5.19 \pm 0.34$ & $6.04 \pm 1.08$ & $5.05 \pm 0.23$ & $1290 \pm 70$ \\
\hline 40 & $-0.33 \pm 0.01$ & $0.20 \pm 0.01$ & $5.43 \pm 0.28$ & $5.17 \pm 1.17$ & $6.10 \pm 0.32$ & $1490 \pm 80$ \\
\hline 70 & $-0.28 \pm 0.01$ & $0.17 \pm 0.01$ & $7.02 \pm 0.43$ & $2.24 \pm 1.08$ & $6.57 \pm 0.34$ & $1680 \pm 80$ \\
\hline 100 & $-0.27 \pm 0.01$ & $0.15 \pm 0.01$ & $9.50 \pm 0.42$ & $0.68 \pm 0.42$ & $8.59 \pm 0.57$ & $1970 \pm 130$ \\
\hline 130 & $-0.27 \pm 0.02$ & $0.17 \pm 0.02$ & $6.89 \pm 0.85$ & $2.80 \pm 0.71$ & $6.55 \pm 0.45$ & $1650 \pm 110$ \\
\hline
\end{tabular}


cylinder bore coatings [25]. The gray scale of the achieved CT data set was chosen to obtain a high contrast to noise ratio such that iron shows highest possible range of contrast within the image, while air as well as aluminum appear to be black in the data. Hence, the quantitative image analysis may not be able to distinguish between air and aluminum and both materials will be detected as porosity. Further on, due to time and sample size limitations, lowest voxel size of $4 \mu \mathrm{m}$ could be obtained in this case. The associated structural resolution does not allow for a re liable identification of all laterally expanded splat interfaces, which typically gap opens only a few micrometers. However, CT data have the advantage being truly three dimensional, while optical microscopy images provide two dimensional information only. Since the latter can reach structural resolutions of less than $1 \mu \mathrm{m}$, both techniques com plement each other in the microstructural characterization of thermally sprayed cylinder bore coatings. While each single image of the CT data is processed by the analysis algorithm individually, mean porosity and its' standard deviation of each measurement position are calculated based on the two dimensional image stack.

Further, CT data is used to measure the coating thickness. Due to the roughened substrate, the material thickness of the coating is defined as the mean material volume over the observed measurement area. Due to the comparably large measurement area of $5 \times 5 \mathrm{~mm}^{2}$, lateral local variations of the cylinder coating in the length scale of $200 \mu \mathrm{m}$ (Fig. 1) will be averaged. Since TWI measurements are averaged on a com parable length scale (Section 3.2), lateral local variations of the cylinder coating are also averaged within the thermal diffusivity measurements. The corresponding $3 \sigma$ interval of thickness measurements via CT data is specified to $2.2 \mu \mathrm{m}$ [25].

\section{Results}

\subsection{TWI measurements}

Selected amplitude and phase images of TWI measurements over the entire excitation frequency spectrum can be found in Fig. 4. The ac quired amplitudes of the TWI measurements strongly decrease with increasing excitation frequency. Further, bright spots within the am plitude images reveal surface contamination of the cylinder coating or contamination of the optical system. Horizontal artifacts within the amplitude images as well as a diffuse artifact at the right edge of the excited area can be identified as reflections of the optical components and the mirror attachments on the highly reflective coating. However, such reflections can be traced back to the phase images and have an impact on the acquired phase values. Since the evaluated area of $6.7 \mathrm{~mm}$ diameter is comparably large to the artifacts, the error of these artifacts on the averaged phase value of the coating will be neglected. The in fluence of lateral heat diffusion on phase values can be found in the respective phase image at $f=5 \mathrm{~Hz}$. While a bright circle along the edge of the excited area can be seen in the phase image, evaluated phase values within the area of $6.7 \mathrm{~mm}$ diameter will not be affected by such lateral heat diffusion processes. Further, in order of variable thicknesses due to the roughened substrate, phase values may represent such lateral thickness variations within the acquired images. However, since the spatial resolution of the infrared system of about $0.2 \mathrm{~mm}$ is in the same order as the lateral thickness variations, such influences cannot be found in the acquired phase images. In addition, the phase images also show a strong decay of the acquired phase values with increasing fre quency according to Eq. 5. Also the signal to noise ratio of the phase images decreases towards higher frequencies. As amplitude images have already revealed, the amplitude decreases towards higher fre quencies resulting in a lowered signal to noise ratio of the phase images. According to the used camera frame rate of $600 \mathrm{~Hz}$, higher ex citation frequencies consequence a lower amount of data points per period, increasing the uncertainty of the applied FFT to the measure ment data.

Calculated fit values $R$ and $\eta$ resulting from the TWI measurements of thermally sprayed coatings at different crankcase positions are shown in Table 1. Furthermore, the table includes thermal diffusivities of the measured samples, while corresponding thickness values were measured with $\mathrm{X}$ ray tomography. The thermal diffusivity values $\alpha$ of the cylinder coating show a strong dependence on the measurements position in the crankcase. An increase of $\alpha$ by $83 \%$ ranging from $5.19 \mathrm{~mm}^{2} / \mathrm{s}$ to $9.50 \mathrm{~mm}^{2} / \mathrm{s}$ is observed comparing TWI measurement at positions $10 \mathrm{~mm}$ and $100 \mathrm{~mm}$. Measurements at positions $40 \mathrm{~mm}$ and $70 \mathrm{~mm}$ underline the increase of the thermal diffusivity of the coating along the cylinder liner. However, a strong decrease occurs towards the lowest section of the liner from position $100 \mathrm{~mm}$ to $130 \mathrm{~mm}$. The mea sured thermal diffusivity value of $6.89 \mathrm{~mm}^{2} / \mathrm{s}$ at position $130 \mathrm{~mm}$ is comparable to the measured value at $70 \mathrm{~mm}$. This trend is visualized in Fig. 5. TWI measurements were performed for three randomly selected engines of the series production for all six cylinder liners at the same angle position. The resulting uncertainties of TWI measurements are calculated from the standard deviation for the corresponding mea surements. Corresponding thermal diffusion lengths based on the measured thermal diffusivities range between $105 \mu \mathrm{m}(150 \mathrm{~Hz})$ and $777 \mu \mathrm{m}(5 \mathrm{~Hz})$, while CT measurements have obtained material thickness values ranging from $235 \mu \mathrm{m}$ to $275 \mu \mathrm{m}$. Hence, lower excitation fre quencies create thermal waves with thermal diffusion lengths larger

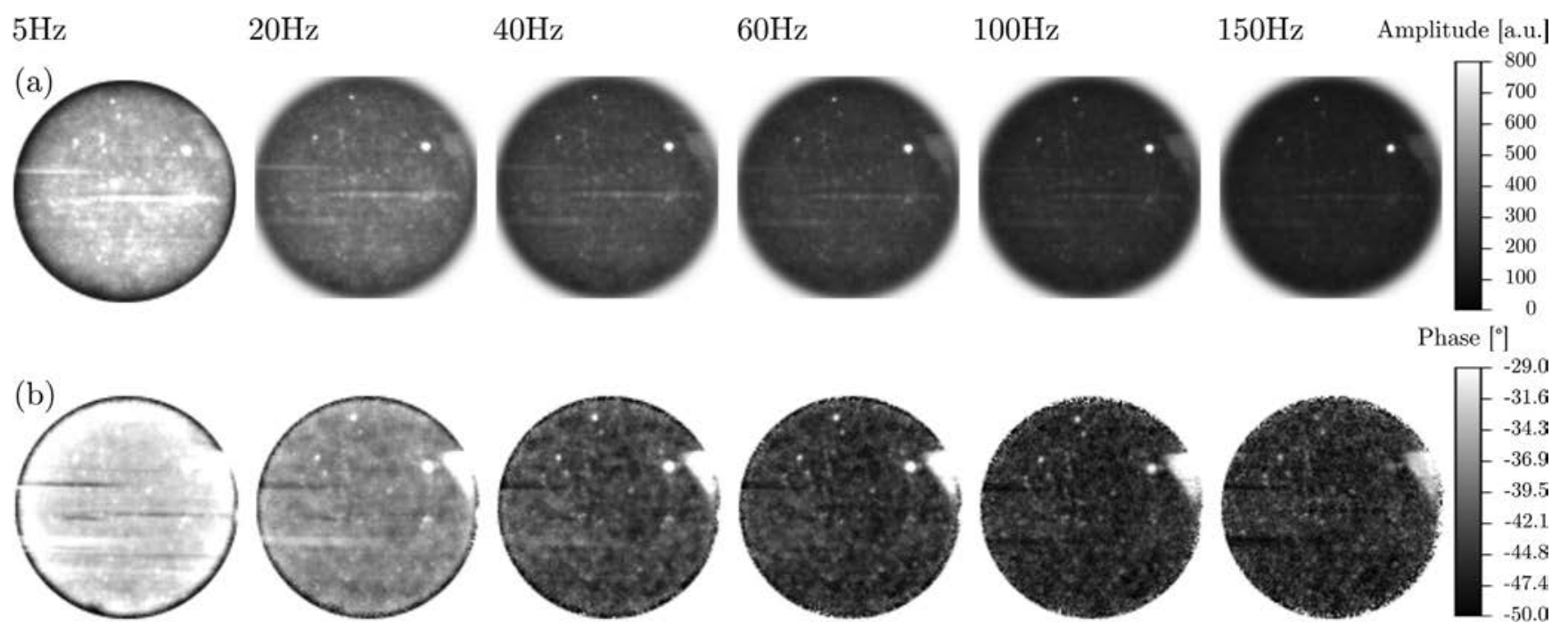

Fig. 4. (a) Amplitude and (b) phase images of TWI measurement at the depicted excitation frequencies for one randomly selected measurement position. 


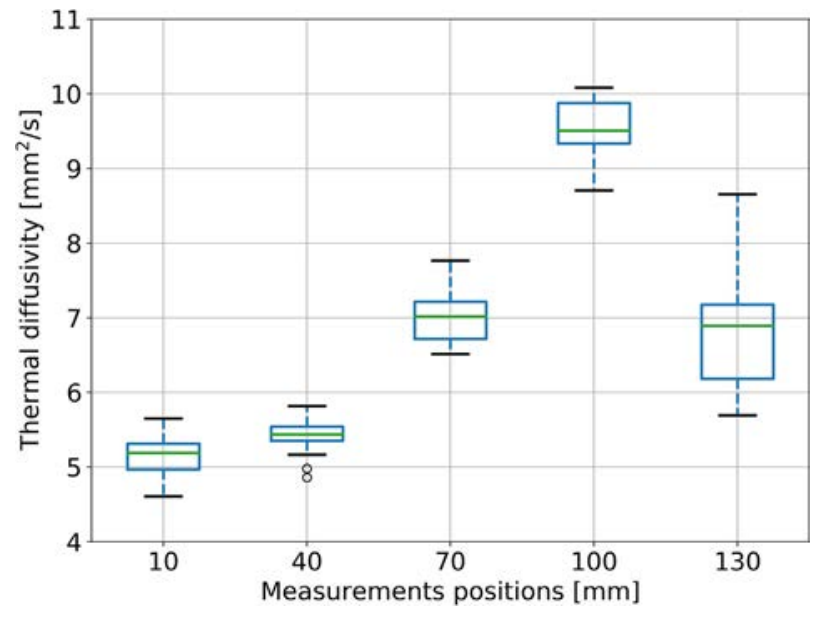

Fig. 5. Thermal diffusivity values of the cylinder coating along the crankcase positions for the evaluated three engines. The green lines indicate the median of the underlying data; the boxes represent the upper \& lower quartile; the whiskers are limited to $1.5 \times$ the interquartile range. Values outside the whiskers are shown as circles.

than the material thickness of the coating, while higher frequencies are used to reach saturation of Eq. (5).

The measured values of the reflection coefficient $R$ show a com parable behavior to the thermal diffusivities. A distinct increase in $R$ downwards the cylinder liner occurs, while $R$ peaks at $100 \mathrm{~mm}$ and re mains constant towards $130 \mathrm{~mm}$ underneath the cylinder head sealing surface.

Both, thermal diffusivity $\alpha$ as well as reflection coefficient $R$ indicate varying thermal and hence microstructure of the thermally sprayed cylinder bore coating $[5,18]$. These results are further analyzed with cross sections and porosity analysis.

\subsection{Cross sections}

Respective optical microscopy images of the cross sections taken at comparable positions as TWI measurements are shown in Fig. 7 (a), while the segmented lateral splat interfaces are shown in Fig. 7 (b). The cross sections show strong dependence of the amount and shape of lateral splat interface on the position in the crankcase. Cross section images taken at $10 \mathrm{~mm}$ and $40 \mathrm{~mm}$ depict enhanced lateral splat inter faces, while the detected splat interfaces decrease towards the inter mediate position $70 \mathrm{~mm}$ and especially cross sections at $100 \mathrm{~mm}$ do not show any pronounced lateral splat interfaces within the image. How ever, regarding to the lowest measurement position $130 \mathrm{~mm}$, lateral splat interfaces are again visible in the illustrated cross section image comparable to position $70 \mathrm{~mm}$. While segmented splat interfaces can be found within the entire thermally sprayed cylinder bore coating at the respective positions, further binding errors can also be observed along the roughening profile of the crankcase substrate. While such binding errors of the coating material to the crankcase substrate can hardly be seen in the microscopy images, the segmentation algorithm strongly increases the ability to detect such errors (Fig. 7, dotted boxes).

Using the segmentation algorithm to obtain quantitative analysis from the cross section images, the observed area of lateral splat inter faces is illustrated in Fig. 6 . The calculated splat percentage show a strong, almost linear decrease from position $10 \mathrm{~mm}$ over $40 \mathrm{~mm}$ and $70 \mathrm{~mm}$ towards $100 \mathrm{~mm}$ of about $87 \%$, which is followed by an increase towards $130 \mathrm{~mm}$ to a comparable level as position $70 \mathrm{~mm}$. Lowest standard deviation can be found at $100 \mathrm{~mm}$, while especially $10 \mathrm{~mm}, 40 \mathrm{~mm}$ and $70 \mathrm{~mm}$ show comparable results.

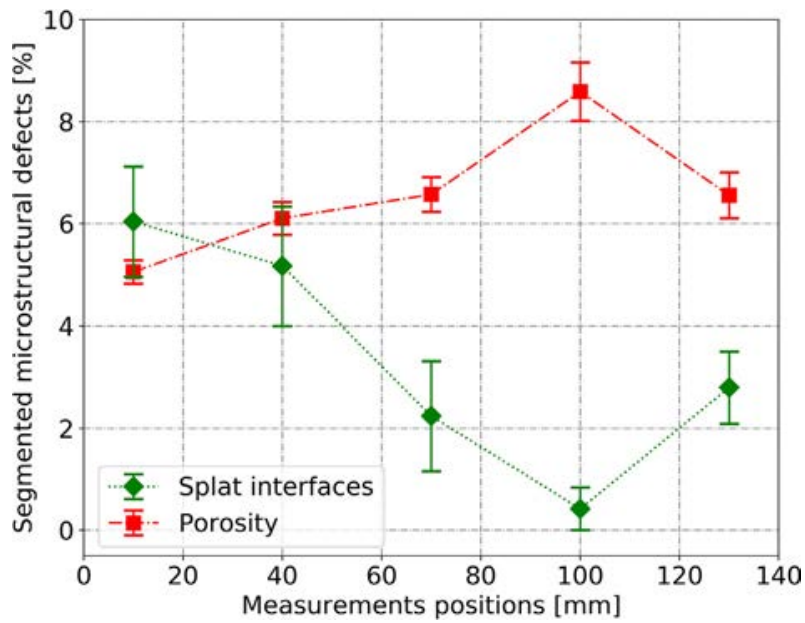

Fig. 6. Detected area of lateral splat interfaces in the cross section images values compared to the mean detected porosity in the CT data of the cylinder coating along the crankcase positions. All shown uncertainties represent the standard deviation of the analyzed cross section images and CT data at the depicted measurement positions.

\subsection{X ray computed tomography}

Acquired CT data of the cylinder coating at crankcase position $100 \mathrm{~mm}$ is shown in Fig. 8. Coating porosity can be seen in all images, while Fig. 8 (d) also shows parts of the roughened substrate. In contrast to the optical microscopy images of the cross sectional specimens in Fig. 7, no laterally expanded splat interfaces may be found in the CT data due to the lower image resolution. However, the 3D CT data set allows to obtain quantitative analysis of the porosity distribution within the cylinder coating. The evaluated porosity distribution within the coating material at different crankcase positions is illustrated in Fig. 9. The porosity distribution within the coating material varies with measurement position along the cylinder liner. Highest porosity is found at $100 \mathrm{~mm}$, while positions $40 \mathrm{~mm}, 70 \mathrm{~mm}$ and $130 \mathrm{~mm}$ show comparable value levels. The lowest porosity of the coating material appears at $10 \mathrm{~mm}$. All curves reach a constant porosity level at coating depth between $20 \mu \mathrm{m}$ and $120 \mu \mathrm{m}$ underneath the cylinder coating sur face, while the strong decrease of the porosity below $20 \mu \mathrm{m}$ is assigned to mechanical processing of the thermally sprayed coatings. This is supported by the fact that a low percentage of porosity appears close to the surface in combination with only a few but relatively large pores. Hence, the coating is compressed by the mechanical processing, while single splat interfaces are ripped open, resulting in a smooth, hard surface with oil reservoirs. CT parameters were chosen to gain a high contrast to noise ratio of the iron based coating material. However, this parametrization reduces implicitly the contrast to noise ratio of air as well as aluminum based substrate material and hence reduces the segmentation ability of those materials. Therefore, the segmentation algorithm separates coating material from any other material without proper segmentation of air and aluminum substrate. Hence, a de creasing amount of coating material at depths of about $120 \mu \mathrm{m}$ under neath the cylinder running surface is not connected to rising porosity, but to the beginning substrate material (Fig. 8(d)). Thus, an in depth region between about $20 \mu \mathrm{m}$ and $120 \mu \mathrm{m}$ of the coating is identified, which contains only coating material and air in the form of inner vo lume defects. This region is reliably segmented by the above described processing chain. The average porosity of the described coating depths ranging from $20 \mu \mathrm{m}$ to $120 \mu \mathrm{m}$ for different crankcase positions can be found in Fig. 6. Further, the average pore size is shown in Table 1 as well as in Fig. 10. Here, mean pore sizes correlate with the observed percentages of porosity such that positions with increased porosity also show increased average pore size and vice versa. CT measurements 

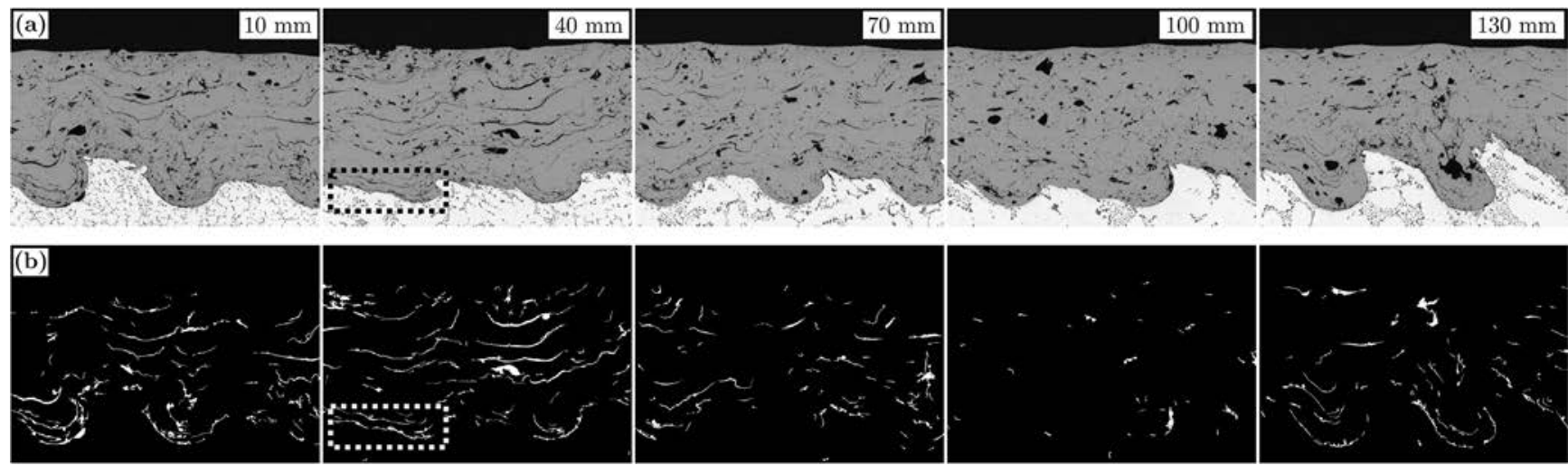

Fig. 7. (a) Optical microscopy images of the thermally sprayed coating cross sections at positions $10 \mathrm{~mm}, 40 \mathrm{~mm}, 70 \mathrm{~mm}, 100 \mathrm{~mm}$ and $130 \mathrm{~mm}$. (b) Segmented lateral splat interfaces of the cross section images. The amount and area of segmented splat interfaces show a strong dependence on the position in the crankcase. Dotted boxes: binding errors and gaps between the coating and the substrate can hardly be seen in the microscopy images, while the segmentation algorithm strongly increases the visibility of such errors.

show mean pore sizes ranging from about $2000 \mu \mathrm{m}^{3}$ at $100 \mathrm{~mm}$, while lowest pore sizes of around $1300 \mu \mathrm{m}^{3}$ can be found at $10 \mathrm{~mm}$. All shown uncertainties represent the standard deviation summarizing evaluated CT data ranging from $20 \mu \mathrm{m}$ to $120 \mu \mathrm{m}$.

\section{Discussion}

Thermal diffusivity as well as lateral splat interfaces and porosity distribution of the cylinder coatings indicate strong dependencies on the measurement position within the crankcase liner. This leads to the assumption, that the investigated properties may correlate with one another but also depend on the NANOSLIDE ${ }^{\mathrm{TM}}$ process chain. As seen in Fig. 5 and 6, crankcase positions with enhanced thermal diffusivity show lowered percentages of lateral splat interfaces within the coating along the liners. Consequently, a direct correlation of the thermal dif fusivity to the microstructural properties of the thermally sprayed cy linder bore coatings may be assumed. As Schilder et. al [3] presented in their work, the occurrence of lateral splat interfaces depends on process parameters of the twin wire arc spraying. Since the shape of the crankcase, Nitrogen gas flow, extraction velocity and other process parameters cause turbulences within the crankcase, the Oxygen content

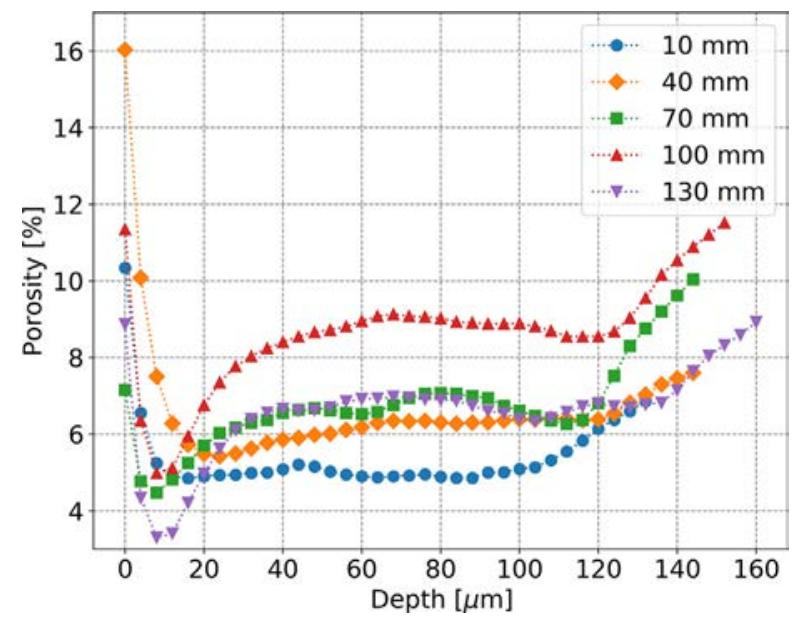

Fig. 9. Coating porosity for different measurement position along the crankcase liner within the layer starting at the surface of the coating towards the roughened substrate interface.
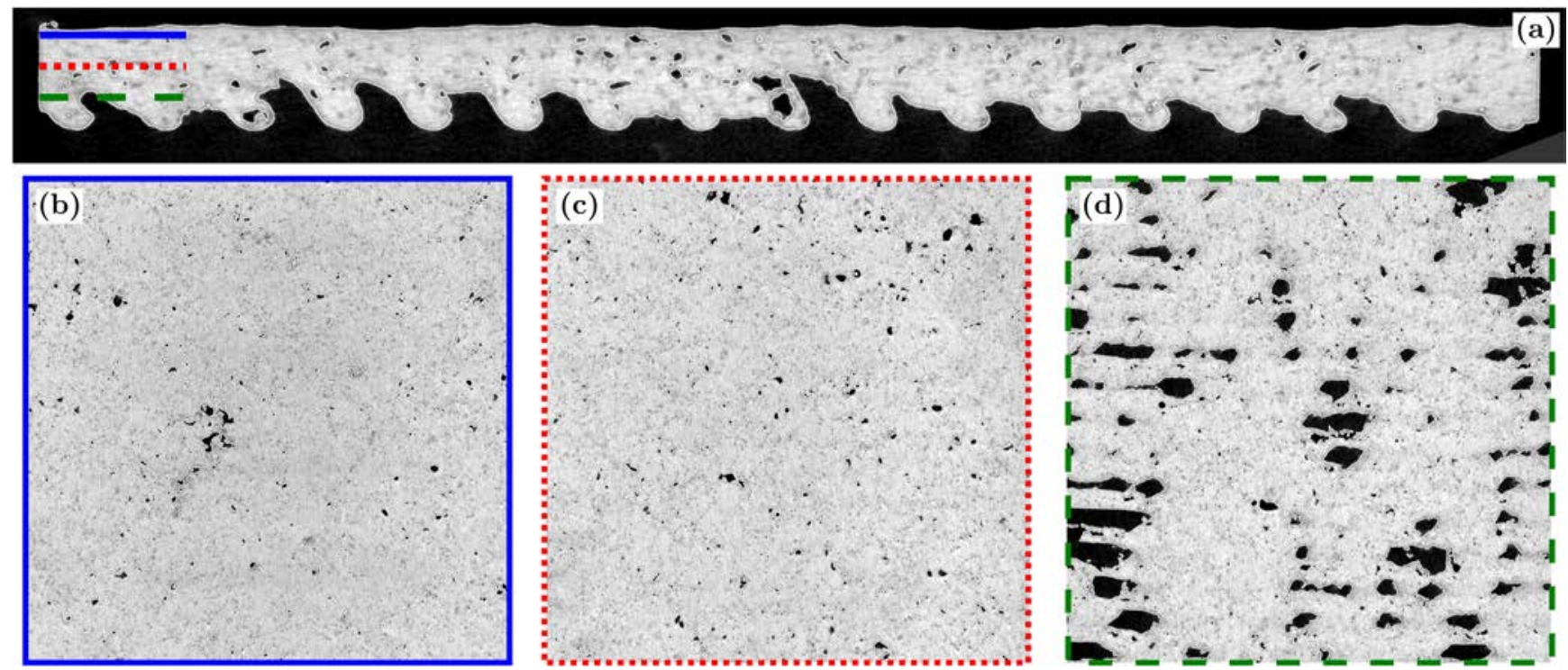

Fig. 8. CT images of the cylinder coating at crankcase position $100 \mathrm{~mm}$. (a) Cross section image of the cylinder coating including the roughened substrate. (b) - (d) CT images parallel to the surface at coating depths of $20 \mu \mathrm{m}, 70 \mu \mathrm{m}$ and $140 \mu \mathrm{m}$ respectively. In (d), beginning roughening structure of the aluminum-based substrate can be observed. 


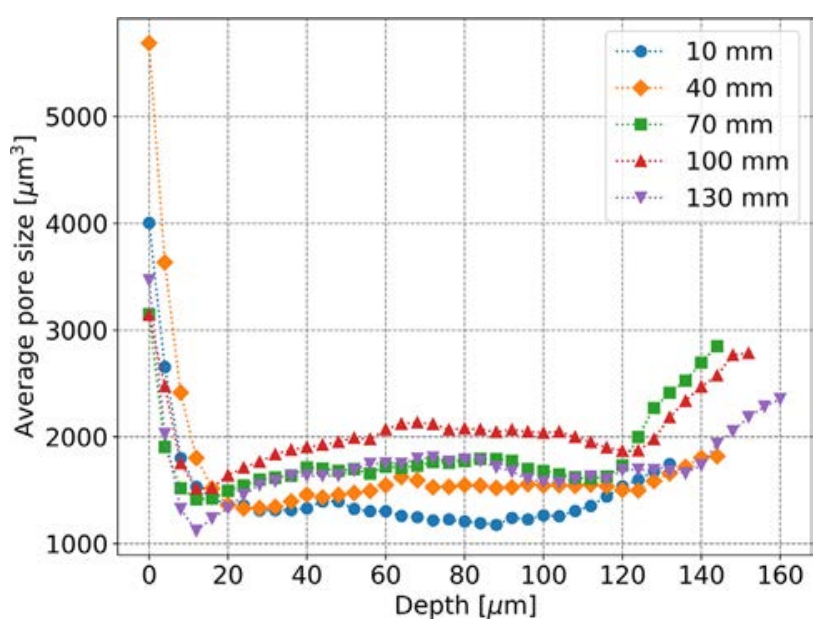

Fig. 10. Average pore size for different measurement position along the crankcase liner within the layer starting at the surface of the coating towards the roughened substrate interface.

of the spray jet depends on the burner position in the crankcase. Schilder et al. [3] used computational fluid dynamics (CFD) simulations as well as coating experiments to compare the Oxygen mass fraction at crankcase positions $20 \mathrm{~mm}$ and $100 \mathrm{~mm}$. They found a significantly higher oxygen percentage at position $20 \mathrm{~mm}$ compared to $100 \mathrm{~mm}$. The enhanced Oxygen content at burner position $20 \mathrm{~mm}$ leads to distinct oxidation of the spraying particles and consequences a significant in crease of splat interfaces within the coating material [3,4]. These ob servations are covered by the presented results in this paper, as it is illustrated in Fig. 7.

Combining thermal diffusivity measurements with the lateral splat interface analysis of the cross section images as well as the knowledge of the influence of process parameters on the microstructure of the coating, the local dependence of the thermal diffusivity can be directly related to the lateral splat interfaces of the cylinder coating and con sequently with the NANOSLIDE ${ }^{\mathrm{TM}}$ process chain. Due to the shape and orientation of the lateral splat interfaces, these defects build up multiple thermal barriers within the cylinder coating and hence lower the thermal diffusivity from the surface towards the substrate interface [18]. In absence of such lateral splats as depicted at position $100 \mathrm{~mm}$, the thermal diffusivity is about $83 \%$ higher than in the upper parts of the cylinder crankcase. Further on, an increase of lateral splat interfaces from measurement position $100 \mathrm{~mm}$ to $130 \mathrm{~mm}$ also results in a decrease of the thermal diffusivity of the cylinder coating back to the level of position $40 \mathrm{~mm}$ and stresses the described observations from the upper measurement positions. Further on, besides enhanced lateral splat in terfaces found at positions $10 \mathrm{~mm}, 40 \mathrm{~mm}$ and $130 \mathrm{~mm}$, also binding er rors and gaps between the coating material and the crankcase substrate can be observed in the respective segmentation images. Regarding to the adhesion mechanism of the coating to the substrate and the desired macro and microstructures, such binding errors may lead to reduced adhesion of the coating, which influences the durability of the com bustion engine as a whole $[3,18]$.

Additionally, CT data is used to analyze the three dimensional dis tribution of spherical like pores within coatings in comparison with their thermal diffusivity at the very same positions. In contrast to other publications $[5,26]$, the porosity distribution as well as the average pore size follows the thermal diffusivity along the crankcase positions such that positions with high thermal diffusivity also show high por osity values as well as high pore sizes and vice versa (Fig. 5 and 6). In general, higher porosity within the thermally sprayed material should lower the thermal conductivity and hence the thermal diffusivity $[5,26]$. Therefore, the percentage of spherical pores may not be the dominant effect influencing the thermal diffusivity, but the shape shows a significant influence. Despite that the porosity percentage excels the lateral splat interface percentage within the cylinder coating (Table 1), the shape and orientation of lateral interfaces compared to spherical pores show the major impact at influencing the thermal diffusivity of the thermally sprayed coatings. Long, laterally expanded binding errors may have a large projected area orthogonal to the cross sectional views observed in this work. Hence, they show a significantly higher influence on the thermal diffusivity than spherical pores without a distinct or ientation and with a smaller projected area.

\section{Conclusion}

Three different types of measurement techniques were used to evaluate thermal and mechanical properties of twin wire arc spray cylinder coatings within three crankcases of combustion engines type OM656 (Mercedes Benz, Stuttgart, Germany). Thermal wave inter ferometry was used to obtain thermal diffusivity measurements at dif ferent positions along the cylinder liner of a combustion engine, while optical microscopy images of cross sections as well as 3D CT data were used to evaluate binding defects of the the thermally sprayed material.

Both, thermal and microstructural properties of thermally sprayed cylinder bore coatings change significantly along the cylinder liner. Microstructural properties directly influence the thermal diffusivity value of the cylinder coating, while especially laterally orientated splat interfaces appear to be dominate at influencing the heat conduction in the material. Lateral splat interfaces build up multiple thermal barriers lowering the thermal diffusivity from surface to substrate, while the absence of the described defects consequences an increase in the thermal diffusivity of more than $80 \%$. The observed correlation opens the ability to evaluate the microstructure of the cylinder coating non destructively and contactless by measuring the thermal diffusivity. However, due to the integral measurement of the thermal diffusivity and the comparably low spatial resolution of $0.2 \mathrm{~mm} / \mathrm{px}$ of the infrared camera in contrast to the observed coating defects, TWI does not allow to resolve such binding defects of the coating microstructure. However, since the microstructure influences the mechanical properties of the cylinder bore coating such as adhesion, cohesion or corrosion re sistance, TWI measurements may be used to quantitatively investigate such mechanical properties non destructively. Additionally, due to the observation of enhanced binding errors and gaps between the coating material and the crankcase substrate at positions with enhanced lateral splat interfaces, following studies and experiments will address the correlation between the microstructure of thermally sprayed coating and its' adhesion mechanism to the substrate.

Further on, as Schilder et. al have proven in their study, micro structure of thermally sprayed cylinder bore coatings can be directly correlated to the twin wire arc spraying process [3]. While constant process parameters are applied during coating of one cylinder liner, varying Oxygen mass fraction of the spray jet atmosphere depending on the burner position causes the observed variations of the coating mi crostructure along the cylinder liner. Hence, correlation between the coating microstructre and the twin wire arc process as well as corre lation between the coating microstructure and the thermal diffusivity of the coating allows to use TWI as a non destructive testing method for thermally sprayed cylinder bore coatings and further to monitor its' NANOSLIDE $^{\mathrm{TM}}$ process. Due to its' reproducibility, fast measurement times and the possibility for automation of the measurement and eva luation procedure, TWI may be used for quality control of the the NANOSLIDE $^{\mathrm{TM}}$ process chain within the large scale production of com bustion engines.

\section{References}

[1] M. Schmidt, H. Spieth, J. Bauer, C. Haubach, Prozesskette NANOSLIDE, 100 Betriebe für Ressourceneffizienz-Band 1, Springer, 2017, pp. 234-237, , https:// doi.org/10.1007/978-3-662-53367-3. 
[2] J. Schommers, H. Scheib, M. Hartweg, A. Bosler, Reibungsminimierung bei Verbrennungsmotoren, MTZ-Motortechnische Zeitschrift 74 (7-8) (2013) 566-573, https://doi.org/10.1007/s35146-013-0170-y.

[3] B. Schilder, A. Garling, F. Reimer, M. Hamann, R. Joos, J. Hüger, M. Pöhlmann, T. Lampke, Cfd enhanced thermal spray process for coating of cylinder bores of car engines, Conference proceedings ITSC, 2019, pp. 441-449.

[4] S. Deshpande, S. Sampath, H. Zhang, Mechanisms of oxidation and its role in microstructural evolution of metallic thermal spray coatings-case study for ni-al, Surface and Coatings Technology 200 (18-19) (2006) 5395-5406, https://doi.org/ 10.1016/j.surfcoat.2005.07.072.

[5] F. Cernuschi, P. Bison, A. Moscatelli, Microstructural characterization of porous thermal barrier coatings by laser flash technique, Acta Materialia 57 (12) (2009) 3460-3471, https://doi.org/10.1016/j.actamat.2009.03.041.

[6] F. Cernuschi, I. Golosnoy, P. Bison, A. Moscatelli, R. Vassen, H.-P. Bossmann, S. Capelli, Microstructural characterization of porous thermal barrier coatings by ir gas porosimetry and sintering forecasts, Acta materialia 61 (1) (2013) 248-262, https://doi.org/10.1016/j.actamat.2012.09.055.

[7] W. Chi, S. Sampath, H. Wang, Microstructure-thermal conductivity relationships for plasma-sprayed yttria-stabilized zirconia coatings, Journal of the American Ceramic Society 91 (8) (2008) 2636-2645, https://doi.org/10.1111/j.1551-2916.2008 02476.x.

[8] R. Yang, Y. He, Optically and non-optically excited thermography for composites: A review, Infrared Physics \& Technology 75 (2016) 26-50.

[9] W. Parker, R. Jenkins, C. Butler, G. Abbott, Flash method of determining thermal diffusivity, heat capacity, and thermal conductivity, Journal of Applied Physics 32 (9) (1961) 1679-1684, https://doi.org/10.1063/1.1728417.

[10] A. Bento, D. Almond, The accuracy of thermal wave interferometry for the evaluation of thermophysical properties of plasma-sprayed coatings, Measurement Science and Technology 6 (7) (1995) 1022, https://doi.org/10.1088/0957-0233/6/ $7 / 025$

[11] G. Busse, D. Wu, W. Karpen, Thermal wave imaging with phase sensitive modulated thermography, Journal of Applied Physics 71 (8) (1992) 3962-3965, https://doi. org $/ 10.1063 / 1.351366$

[12] X. Maldague, Theory and practice of infrared technology for nondestructive testing, Wiley series in microwave and optical engineering, John Wiley \& Sons Inc, New York, USA, 2001.

13] D. Wu, G. Busse, Lock-in thermography for nondestructive evaluation of materials, Revue générale de thermique 37 (8) (1998) 693-703, https://doi.org/10.1016/ S0035-3159(98)80047-0.
[14] C. Bennett, R. Patty, Thermal wave interferometry: a potential application of the photoacoustic effect, Applied Optics 21 (1) (1982) 49-54, https://doi.org/10.1364/ AO.21.000049.

[15] J. König, M. Lahres, O. Methner, Quality designed twin wire arc spraying of aluminum bores, Journal of Thermal Spray Technology 24 (1-2) (2015) 63-74, https://doi.org/10.1007/s11666-014-0170-6.

[16] P.L. Fauchais, J.V. Heberlein, M.I. Boulos, Coating characterizations, Thermal Spray Fundamentals, Springer, 2014, pp. 1113-1250, https://doi.org/10.1007/978-0387-68991-3.

[17] I. Gedzevicius, A. Valiulis, Analysis of wire arc spraying process variables on coatings properties, Journal of Materials Processing Technology 175 (1-3) (2006) 206-211, https://doi.org/10.1016/j.jmatprotec.2005.04.019.

[18] L. Pawlowski, P. Fauchais, Thermal transport properties of thermally sprayed coatings, International materials reviews 37 (1) (1992) 271-289, https://doi.org/ 10.1179/imr.1992.37.1.271

[19] A. Bendada, M. Lamontagne, H. Roberge, Influence of thermal properties on the sensitivity of thermal wave interferometry for the characterization of plasmasprayed coatings, International Journal of Thermophysics 24 (1) (2003) 207-222, https://doi.org/10.1023/A:1022322517684.

[20] P. Patel, D. Almond, Thermal wave testing of plasma-sprayed coatings and a comparison of the effects of coating microstructure on the propagation of thermal and ultrasonic waves, Journal of Materials Science 20 (3) (1985) 955-966, https:// doi.org/10.1007/BF00585740.

[21] A. Bendada, N. Baddour, A. Mandelis, C. Moreau, Experimental investigation on the reliability of thermal wave interferometry in the thermophysical characterization of plasma sprayed coatings, International Journal of Thermophysics 26 (3) (2005) 881-892, https://doi.org/10.1007/s10765-005-5584-4

[22] D. Almond, P. Patel, I. Pickup, H. Reiter, An evaluation of the suitability of thermal wave interferometry for the testing of plasma sprayed coatings, NDT International 18 (1) (1985) 17-24, https://doi.org/10.1016/0308-9126(85)90038-0.

[23] R. Krankenhagen, M. Ziegler, C. Maierhofer, Systematic errors in the evaluation of uncorrected data from thermographic lock-in measurements.

[24] C. Ronsen, P. Denjiver, Connected Components in Binary Images: The Detection Problem, John Wiley \& Sons Inc, New York, USA, 1984

[25] S. Lindemann, Zerstörungsfreie Charakterisierung thermisch gespritzte Zylinderlaufflächen, Ph.D. thesis Universität Magdeburg, 2019.

[26] Y. Huang, N. Hu, Y. Zeng, X. Song, C. Lin, Z. Liu, J. Zhang, Effect of different types of pores on thermal conductivity of ysz thermal barrier coatings, Coatings 9 (2) (2019) 138, https://doi.org/10.3390/coatings9020138. 
Karlsruher Institut für Technologie

\section{Repository KITopen}

Dies ist ein Postprint/begutachtetes Manuskript.

\section{Empfohlene Zitierung:}

Arbogast, J.; Schaller, R.; Lindemann, S.; Schilder, B.; Rohde, M.; Seifert, H. J.

Thermal wave interferometry measurements and microstructural analysis of twin wire arc spray cylinder coatings for passenger car engines. 2020. Infrared physics \& technology, 105.

doi: $\underline{10.5445 / I R / 1000106015}$

Zitierung der Originalveröffentlichung:

Arbogast, J.; Schaller, R.; Lindemann, S.; Schilder, B.; Rohde, M.; Seifert, H. J. Thermal wave interferometry measurements and microstructural analysis of twin wire arc spray cylinder coatings for passenger car engines. 2020. Infrared physics \& technology, 105, 103216.

doi:10.1016/j.infrared.2020.103216 